RESEARCH ARTICLE

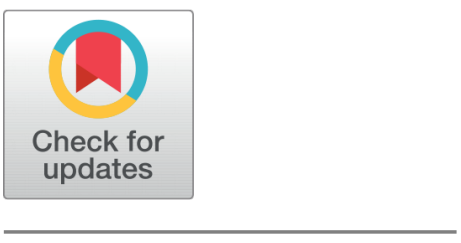

open acCess

Received: 18.07.2021

Accepted: 23.09.2021

Published: 27.10 .2021

Citation: Vembarasi G, Gowri R (2021) Super Root Cube of Cube Difference Labeling of Some Special Graphs. Indian Journal of Science and Technology 14(35): 2778-2783. https://doi.org/

10.17485/IJST/v14i35.1336

* Corresponding author.

vembagopal@gmail.com

Funding: None

Competing Interests: None

Copyright: ๑ 2021 Vembarasi \& Gowri. This is an open access article distributed under the terms of the Creative Commons Attribution License, which permits unrestricted use, distribution, and reproduction in any medium, provided the original author and source are credited.

Published By Indian Society for Education and Environment (iSee)

ISSN

Print: 0974-6846

Electronic: 0974-5645

\section{Super Root Cube of Cube Difference Labeling of Some Special Graphs}

\author{
G Vembarasi ${ }^{1 *}$, R Gowri ${ }^{2}$ \\ 1 Research Scholar, Department of Mathematics, Government College for Women \\ (Autonomous),, Kumbakonam, India \\ 2 Assistant professor, Department of Mathematics, Government College for Women \\ (Autonomous), Kumbakonam, India
}

\section{Abstract}

Background/Objectives: This study gives an extended and the new kinds of super root cube of cube difference labeling of some graphs are obtained. Methods/ Findings: We derive super root cube of cube difference labeling of path related graph and analyzed cycle related graphs.

Keywords: Triangular Snake T_n; Cycle graph C_n; Crown C_n@K_1; pendent edge to both sides of each vertex of a path $\mathrm{Pn}$; supr root cube of cube difference labeling of graphs.

\section{Introduction}

All graphs $G=(V(G), E(G))$ with p vertices and q edges we mean a simple connected and undirected graph. In 2012, J. Shiama ${ }^{(1)}$, studied square difference labeling of some graphs. In 2013, J. Shiama ${ }^{(2)}$, introduced the concept of cube difference labelings and investigated the labelings for certain graphs. S.Sandhya et.al ${ }^{(3)}$, was initiated the concept of root square mean labeling of graphs. In 2016, M. Kannan et.al ${ }^{(4)}$, introduced the concept of super root square mean labeling of disconnected graphs are discussed. In 2017, R.Gowri and G.Vembarasi ${ }^{(5)}$, was discussed root cube mean labeling of graphs. R.Gowri and G.Vembarasi ${ }^{(6)}$, extended the new concept of root cube difference labeling of graphs are introduced in 2018. In 2019, S.Kulandhai Theresa and K.Romila ${ }^{(7)}$, was discussed the concept of cube root cube mean labeling of graphs are introduced. In 2020, R.Gowri and G.Vembarasi ${ }^{(8)}$ recently introduced the concept of root cube of cube difference labeling of graphs. Likewise, many authors have discussed this topic in their work. In this study we discuss about the super root cube of cube difference labeling and investigate certain families of graphs.

\section{Preliminaries}

\section{Definition 2.1(5)}

The graph obtained by joining a simple pendent edge to each vertex of a path is called a Comb graph.

\section{Definition $2.2^{(5)}$}

A walk in which vertices are called a path. A path on $n$ vertices is denoted by $\mathrm{P}_{n}$. 


\section{Definition $2.3^{(5)}$}

The product graph $P_{2} \times P_{n}$ is called a Ladder and it is denoted by $L_{n}$.

\section{Definition $2.4^{(9)}$}

A graph $\mathrm{G}$ with $\mathrm{p}$ vertices and q edges then $f: V(G) \rightarrow\{1,2, \ldots, p+q\}$ be an injective function. For each edge e=uv. Let $f^{*}(e=$ $u v)=\left[\sqrt{\frac{f(u)^{2}+f(v)^{2}}{2}}\right]$ (or) $\left[\sqrt{\frac{f(u)^{2}+f(v)^{2}}{2}}\right]$ then $\mathrm{f}$ is called a super root square mean labeling if $f(v) \cup\left\{f^{*}(e): e \in E(G)\right\}=$ $\{1,2, \ldots, p+q\}$. A graph that admits a super root mean labeling is called a super root mean graph.

\section{Definition $2.5^{(2)}$}

Let $G=(V(G), E(G))$ be a graph. $\mathrm{G}$ is said to be a cube difference labeling if there exists a injective function $f: v(G) \rightarrow$ $\{0,1, \ldots, p-1\}$ such that the induced function $f^{*}: E(G) \rightarrow N$ is given by $f^{*}(u v)=\left|[f(u)]^{3}-[f(v)]^{3}\right|$ is injective.

\section{Definition $2.6^{(6)}$}

Let $G=(V(G), E(G))$ be a graph. $\mathrm{G}$ is said to be a cube difference labeling if there exists a injective function $f: v(G) \rightarrow\{0,1, \ldots, p-1\}$ such that the induced function $f^{*}: E(G) \rightarrow N$ is given by $f^{*}(u v)=$ $\left[\sqrt{\mid[f(u)]^{3}-\left[f(v)^{3}\right]} \mid\right.$ (or) $\left[\sqrt{\left|[f(u)]^{3}-[f(v)]^{3}\right|}\right]$ is injective.

\section{Super Root Cube Of Cube Difference Labeling of Graphs}

\section{Definition 3.1}

A graph $\mathrm{G}$ with p vertices and q edges then $f: V(G) \rightarrow\{1,2, \ldots, p+q\}$ be an injective function. For each edge $\mathrm{e}=u v$. Let $f^{*}(e=u v)=\left[\sqrt{\left|\left(f(u)^{3}-f(v)^{3}\right)^{\frac{1}{3}}\right|}\right]$ (or) $\left[\sqrt{\left|\left(f(u)^{3}-f(v)^{3}\right)^{\frac{1}{3}}\right|}\right]$, then $\mathrm{f}$ is called a super root cube of cube difference labeling if $f(v) \cup\left\{f^{*}(e): e \in E(G)\right\}=\{1,2, \ldots, p+q\}$. A graph is called a super root cube of cube difference labeling.

\section{Theorem 3.2}

Triangular Snake $T_{n}$ is a super root cube of cube difference labeling of graph.

Proof : A Triangular Snake $T_{n}$ is obtained from a path $u_{1}, u_{2}, \ldots, u_{n}$ by joining $u_{i}$ and $u_{i+1}$ to a new vertex $v_{i}$ for $1 \leq i \leq n$ That is every edge of a path is replaced by a triangular $C_{3}$. Define the function $f: V(G) \rightarrow\{1,2, \ldots, p+q\}$ by

$$
\begin{array}{lllrl}
f\left(u_{i}\right)=2 i, & \text { for } & & 1 \leq i \leq n \\
f\left(v_{i}\right)=2 i-1, & \text { for } & & 1 \leq i \leq n .
\end{array}
$$

And the induced edge labeling function $f^{*}: E(G) \rightarrow N$ defined by

$$
f^{*}(e=u v)=\left[\sqrt{\left|\left(f(u)^{3}-f(v)^{3}\right)^{\frac{1}{3}}\right|}\right]
$$

Then the edge sets are,

$$
\begin{gathered}
f^{*}\left(u_{i} u_{i+1}\right)=\sqrt{\left|\left(24 i^{2}+24 i+8\right)^{\frac{1}{3}}\right|}, \quad \text { for } 1 \leq i \leq n-1 \\
f^{*}\left(u_{n-1} u_{n}\right)=\sqrt{\left|\left(24 n^{2}-24 n+8\right)^{\frac{1}{3}}\right|} \\
f^{*}\left(u_{i} v_{i}\right)=\sqrt{\left|\left(12 i^{2}-6 i+1\right)^{\frac{1}{3}}\right|}, \quad \text { for } 1 \leq i \leq n \\
f^{*}\left(u_{n} v_{n}\right)=\sqrt{\mid\left(12 n^{2}-6 n+1\right)^{\frac{1}{3} \mid}}
\end{gathered}
$$




$$
\begin{gathered}
f^{*}\left(u_{i+1} v_{i}\right)=\sqrt{\mid\left(36 i^{2}+18 i+9\right)^{\frac{1}{3}}} \mid \text { for } 1 \leq i \leq n \\
f^{*}\left(u_{n+1} v_{n}\right)=\sqrt{\left|\left(36 n^{2}+90 n+63\right)^{\frac{1}{3} \mid}\right|}
\end{gathered}
$$

Hence the graph G is a Super root cube of cube difference labeling.

\section{Example 3.3}

Super root cube of cube difference labeling of $T_{4}$ is given below.

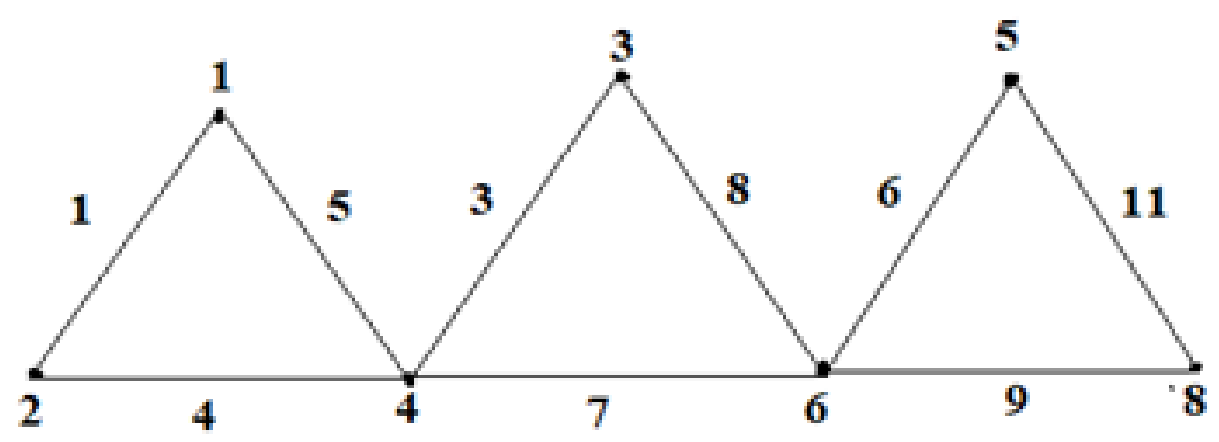

Fig 1.

\section{Theorem 3.4}

The Cycle graph $C_{n}$ is a Super root cube of cube difference labeling.

Proof : A closed path is called a cycle. A cycle on $\mathrm{n}$ vertices is denoted by $C_{n}$ graph with vertices $u_{1}, u_{2}, \ldots, u_{n}$ and the edges $e_{1}, e_{2}, \ldots, e_{m}$ Define the function $f: V(G) \rightarrow\{1,2, \ldots, p+q\}$ by

$$
f\left(u_{i}\right)=i \quad \text { for } 1 \leq i \leq n
$$

And the induced edge labeling function $f^{*}: E(G) \rightarrow N$ defined by

$$
f^{*}(e=u v)=\left[\sqrt{\left|\left(f(u)^{3}-f(v)^{3}\right)^{\frac{1}{3}}\right|}\right]
$$

Then the edges labels are,

$$
\begin{gathered}
f^{*}\left(u_{i} u_{i+1}\right)=\sqrt{\left(3 i^{2}+3 i+1\right)^{\frac{1}{3}}}, \quad \text { for } 1 \leq i \leq n-1 \\
f^{*}\left(u_{n-1} u_{n}\right)=\sqrt{\left(3 n^{2}-3 n+1\right)^{\frac{1}{3}}} \\
f^{*}\left(u_{n} u_{1}\right)=\sqrt{\left(n^{3}-1\right)^{\frac{1}{3}}}
\end{gathered}
$$

Hence the graph $C_{n}$ is a Super root cube of cube difference labeling. 


\section{Example 3.5}

The following is an example for $C_{7}$ is a Super root cube of cube difference labeling of graph.

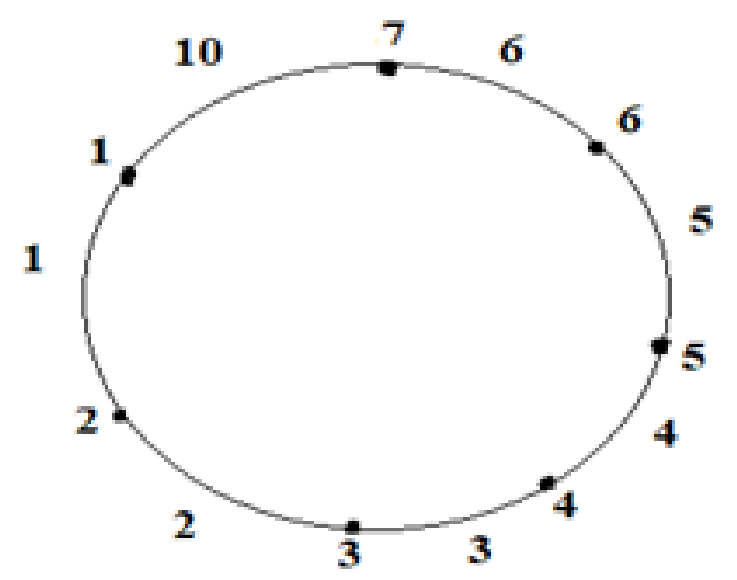

Fig 2.

\section{Theorem 3.6}

The crown $C_{n} \Theta K_{1}$ is a Super root cube of cube difference labeling of graph.

Proof: Let $C_{n}$ be the Cycle $u_{1} u_{2} . . u_{n} u_{1}$ and $v_{i}$ be the pendant vertices adjacent to $u_{i}, 1 \leq i \leq n$. Define the function $f: V\left(C_{n} \Theta K_{1}\right) \rightarrow\{1,2, \ldots, p+q\}$ by

$$
\begin{aligned}
& f\left(u_{i}\right)=i, \\
& \text { for } 1 \leq i \leq n \\
& f\left(v_{i}\right)=i+6, \\
& \text { for } \quad 1 \leq i \leq n .
\end{aligned}
$$

And the induced edge labeling function $f^{*}: E(G) \rightarrow N$ defined by

$$
f^{*}(e=u v)=\left[\sqrt{\left|\left(f(u)^{3}-f(v)^{3}\right)^{\frac{1}{3}}\right|}\right]
$$

Then the edge sets are,

$$
\begin{gathered}
f^{*}\left(u_{i} u_{i+1}\right)=\sqrt{\mid\left(24 i^{2}+24 i+8\right)^{\frac{1}{3} \mid}}, \quad \text { for } 1 \leq i \leq n-1 \\
f^{*}\left(u_{n-1} u_{n}\right)=\sqrt{\left|\left(24 n^{2}-24 n+8\right)^{\frac{1}{3}}\right|} \\
f^{*}\left(u_{i} v_{i}\right)=\sqrt{\left|\left(12 i^{2}-61+1\right)^{\frac{1}{3}}\right|}, \quad \text { for } 1 \leq i \leq n \\
f^{*}\left(u_{n} v_{n}\right)=\sqrt{\left|\left(12 n^{2}-6 n+1\right)^{\frac{1}{3}}\right|} \\
f^{*}\left(u_{i+1} v_{i}\right)=\sqrt{\left|\left(36 i^{2}+18 i+9\right)^{\frac{1}{3}}\right|}, \quad \text { for } 1 \leq i \leq n \\
f^{*}\left(u_{n+1} v_{n}\right)=\sqrt{\left|\left(36 n^{2}+90 n+63\right)^{\frac{1}{3}}\right|}
\end{gathered}
$$

Hence the graph $G$ is a Super root cube of cube difference labeling. 


\section{Example 3.7}

Super root cube of cube difference labeling of $C_{n} \Theta K_{1}$ is given below.

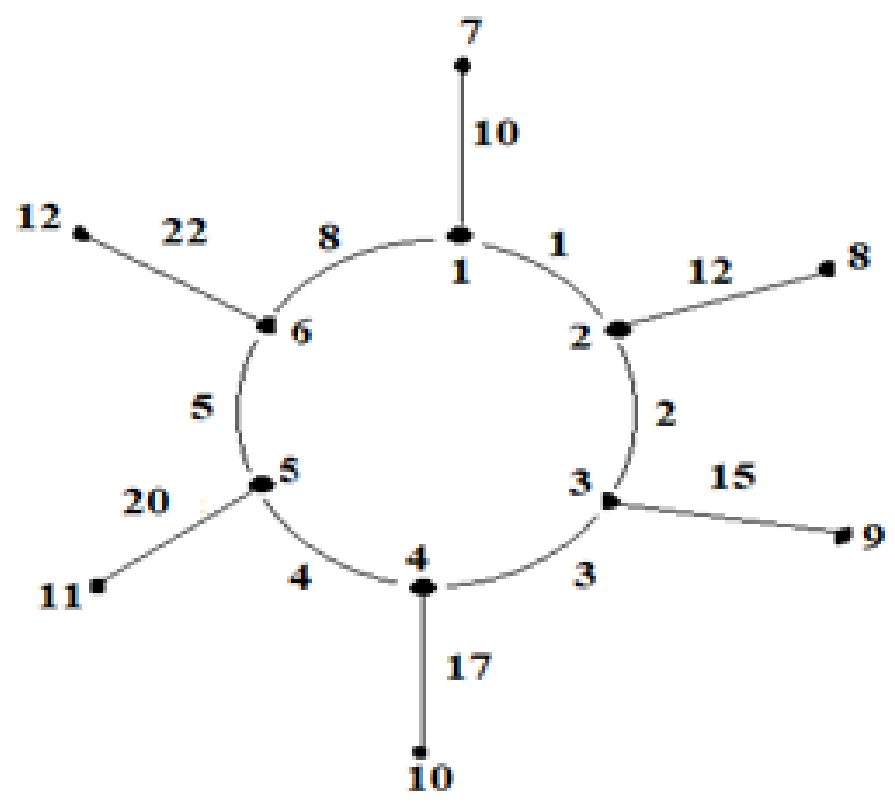

Fig 3.

\section{Theorem 3.8}

Let $\mathrm{G}$ be a graph obtained by attaching a pendant edge to both sides of each vertex of a path $P_{n}$. Then $\mathrm{G}$ is a Super root cube of cube difference labeling of graph only if $n \geq 5$. .

Proof: Let $\mathrm{G}$ be path $P_{n}$. The graph obtained by attaching pendant edges to both sides of each vertex. Let $x_{i}, y_{i}$ and $z_{i}$ for $1 \leq$ $i \leq n$ be the new vertices of $G$. Define the function $f: V(G) \rightarrow\{1,2, \ldots, p+q\}$ by

$$
\begin{aligned}
& f\left(x_{i}\right)=3 i-1 \quad \text { for } 1 \leq i \leq n \\
& f\left(y_{i}\right)=3 i \quad \text { for } 1 \leq i \leq n \\
& f\left(z_{i}\right)=3 i-2 \quad \text { for } 1 \leq i \leq n
\end{aligned}
$$

And the induced edge labeling function $f^{*}: E(G) \rightarrow N$ defined by

$$
f^{*}(e=u v)=\left[\sqrt[1]{\left(f(u)^{3}-f(v)^{3}\right)^{\frac{1}{3}}}\right]
$$

Then the edge sets are,

$$
\begin{gathered}
f^{*}\left(x_{i} x_{i+1}\right)=\sqrt{\left|\left(81 i^{2}+27 i+9\right)^{\frac{1}{3}}\right|}, \quad \text { for } 1 \leq i \leq n-1 \\
f^{*}\left(x_{i} y_{i}\right)=\sqrt{\left|\left(27 i^{2}-9 i+1\right)^{\frac{1}{3}}\right|}, \quad \text { for } 1 \leq i \leq n \\
f^{*}\left(x_{i} z_{i}\right)=\sqrt{\left|\left(27 i^{2}-27 i+7\right)^{\frac{1}{3}}\right|}, \quad \text { for } 1 \leq i \leq n
\end{gathered}
$$

Hence the graph $\mathrm{G}$ is a Super root cube of cube difference labeling. 


\section{Example 3.9}

The graph obtained $P_{5}$ is given below.

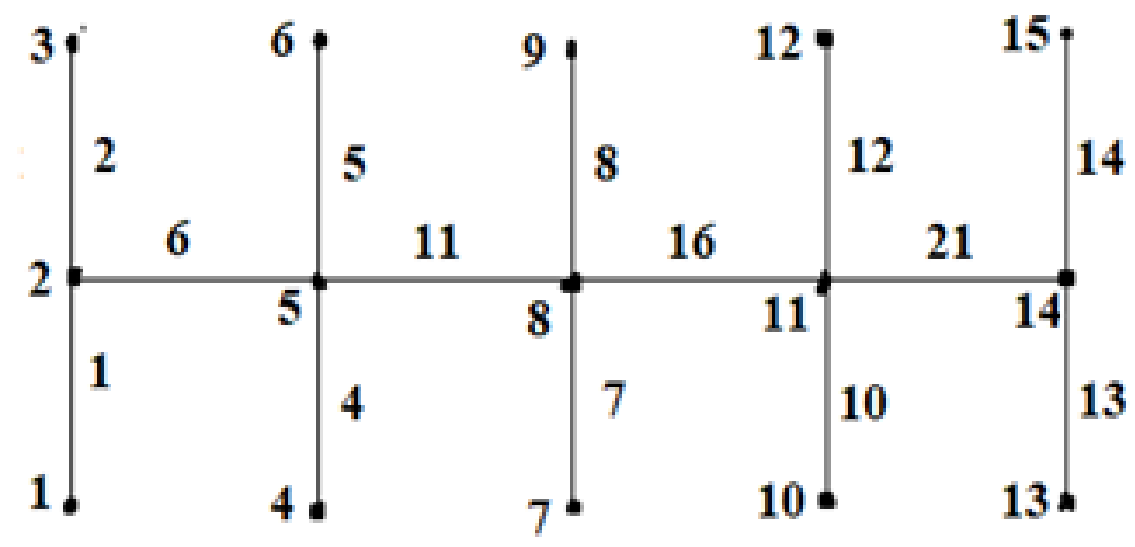

Fig 4.

\section{Conclusion}

In this article we discussed the concept of Super Root Cube of Cube Difference Labeling of Graphs are initiated and also some graphs are introduced and characterized. Then the relative results between path, cycle related graphs are discussed. Here all the edge values are distinct and the resulting edge values do not exceed the vertex value.

\section{References}

1) Shiama J. Square Difference Labeling for Some Graphs. International Journal of Computer Applications. 2012;44(4):30-33. Available from: https: //dx.doi.org/10.5120/6253-8399.

2) Shiama J. Cube Difference Labeling of Some Graphs. International Journal of Engineering Science and Innovative Technology. 2013;2:200-205. Available from: http://www.ijesit.com/Volume\%202/Issue\%206/IJESIT201306_25.pdf.

3) Sandhya SS, Somasundaram S, Anusa S. Root square mean labeling of graphs. International Journal of Contemporary Mathematical Sciences. $2014 ; 9: 667-676$. Available from: https://dx.doi.org/10.12988/ijcms.2014.410105.

4) Kannan M, Prasad R, Gopi R. Super Root Square Mean Labeling of Disconnected Graphs. International Journal of Mathematics and its Applications. 2016;4:93-98.

5) Gowri R, Vembarasi G. Root Cube Mean Labeling of Graphs. International Journal of Engineering Science, Advanced Computing and Bio-Technology. 2017;8(4):248-255. Available from: https://dx.doi.org/10.26674/ijesacbt/2017/49247.

6) Gowri R, Vembarasi G. Root Cube Mean Labeling of Graphs. International Journal of Engineering Science, Advanced Computing and Bio-Technology. 2017;8(4):564-571. Available from: https://dx.doi.org/10.26674/ijesacbt/2017/49247.

7) Therese SK, K R. Cube Root Cube Mean Labeling of Graphs. International Journal of Mathematics Trends and Technology. 2019;65(2):130-135. Available from: https://dx.doi.org/10.14445/22315373/ijmtt-v65i2p520.

8) Gowri R, Vembarasi G. Root Cube of Cube Difference Labeling of Graphs. Strad Research. 2020;7(7):722-729.

9) Thiruganasambandam K, Venkatesan K. Super root square mean labeling of graphs. International Journal of Mathematics and Soft Computing. 2015;5(2):189-195. 